\title{
Primary cutaneous marginal zone lymphomas with plasmacytic differentiation show frequent IgG4 expression
}

\author{
Isabel Brenner ${ }^{1}$, Sabine Roth ${ }^{1}$, Bernhard Puppe ${ }^{1}$, Marion Wobser ${ }^{2}$, Andreas Rosenwald ${ }^{1,3}$ \\ and Eva Geissinger ${ }^{1,3}$
}

${ }^{1}$ Institute of Pathology, University of Würzburg, Würzburg, Germany; ${ }^{2}$ Department of Dermatology, University Clinic Würzburg, Würzburg, Germany and ${ }^{3}$ Comprehensive Cancer Center (CCC) Mainfranken, University and University Hospital, Würzburg, Germany

\begin{abstract}
The expression of IgG4 in malignant B-cell lymphomas has only partially been studied. Recent reports described single cases of marginal zone lymphomas arising in the ocular adnexae that express IgG4. Moreover, a subset of dura-associated marginal zone lymphomas appear to express IgG4 as well. We investigated IgG4 expression in a more systematic manner in a large cohort of marginal zone lymphoma specimens derived from the archive of our institute. Overall, we examined 169 marginal zone lymphomas of various primary sites that displayed a distinct plasmacytic differentiation and light chain restriction, allowing for a detailed investigation of the immunoglobulin heavy chain expression in these tumors by immunohistochemistry. Unexpectedly, primary cutaneous marginal zone lymphomas showed frequent IgG4 expression. Although only 1 out of 120 noncutaneous marginal zone lymphomas, located in the ocular adnexae, expressed IgG4, 19 of 49 (39\%) primary cutaneous marginal zone lymphomas showed this feature, constituting the highest expression rate of IgG4 reported to date in any B-cell lymphoma. None of the IgG4-positive cutaneous marginal zone lymphomas with available clinical data showed evidence of a preexisting systemic IgG4-related disease, suggesting a localized immunologic IgG4-driven pathogenetic process at early stages of the disease. IgG4-positive and IgG4negative primary cutaneous marginal zone lymphomas did not significantly differ in architectural features of the infiltrate or the composition of the reactive T-cell infiltrate as determined by analysis of T-cell content, CD4/CD8 ratio, and content of FOXP3- and PD1-positive T cells. Although the pathogenetic role of IgG4 expression in a significant subset of primary cutaneous marginal zone lymphomas with plasmacytic differentiation remains unclear at present, the demonstration of IgG4 expression in a marginal zone lymphoma involving the skin might be a helpful clue in the routine diagnostic setting, as these tumors will almost invariably be of primary cutaneous origin with an extremely low risk of spread to noncutaneous sites and an excellent prognosis. Modern Pathology (2013) 26, 1568-1576; doi:10.1038/modpathol.2013.106; published online 14 June 2013
\end{abstract}

Keywords: cutaneous lymphoma; IgG4; IgG4-related disease; immunohistochemistry; marginal zone lymphoma

Primary cutaneous B-cell lymphomas encompass primary cutaneous marginal zone lymphomas, primary cutaneous follicle center lymphomas, and primary cutaneous diffuse large B-cell lymphomas, with primary cutaneous marginal zone lymphomas being the second most common entity of this group comprising $\sim 35-40 \%$ of all primary cutaneous B-cell lymphomas. According to the current WHO

Correspondence: Dr E Geissinger, MD, Institute of Pathology, University of Wuerzburg, Josef Schneider-Strasse 2, Wuerzburg, 97080, Germany.

E-mail: geissinger@mail.uni-wuerzburg.de

Received 4 February 2013; revised 3 May 2013; accepted 3 May 2013; published online 14 June 2013 classification, primary cutaneous marginal zone lymphomas are considered extranodal marginal zone lymphomas of the mucosa-associated lymphoid tissue. ${ }^{1}$ They predominantly manifest in adults aged over 40 years, but occasional pediatric cases have been reported as well. Men are affected more frequently than women $(1.5-2: 1){ }^{2}$

Although the etiology and pathogenesis of primary cutaneous marginal zone lymphomas are not well understood, an association with chronic infections, chronic antigen stimulation, and autoimmune diseases has been postulated. Specifically, chronic infection with Borrelia burgdorferi has been associated with primary cutaneous marginal zone lymphomas in Europe, but not in the United States 
or Asia, ${ }^{1-3}$ thus establishing a potential link between an infectious agent and lymphoma development. Occasional cases of primary cutaneous marginal zone lymphomas have also been reported in the context of other chronic inflammatory stimuli, such as vaccinations, tattoos, or radiation therapy. ${ }^{2,4,5}$

Morphologically, primary cutaneous marginal zone lymphomas are characterized by a diffuse or nodular lymphoid infiltrate in the dermis exhibiting a subepidermal grenz zone and often extending into the subcutaneous tissue. The infiltrate often consists of reactive lymph follicles that are surrounded by a conspicuously enlarged marginal zone that contains variable amounts of small to medium-sized B cells, sometimes with abundant pale cytoplasm. Frequently, primary cutaneous marginal zone lymphomas show a lymphoplasmacytoid and plasmacytic differentiation that is particularly evident at the periphery of the infiltrate, for example, in subepidermal and periadnexal areas. ${ }^{1}$ In primary cutaneous marginal zone lymphomas with plasmacytic differentiation, monoclonal immunoglobulin light chain expression $(\kappa$ and $\lambda$ ) as well as immunoglobulin heavy chain expression (IgA, IgE, IgG, and IgM) can be typically visualized by immunohistochemistry. Interestingly, primary cutaneous marginal zone lymphomas preferentially express IgG, IgA, or IgE, in contrast to noncutaneous marginal zone lymphomas that predominantly express IgM, ${ }^{6-9}$ suggesting specific pathogenetic features for primary cutaneous marginal zone lymphomas. This concept is supported on the genetic basis, as translocations involving API2 and BCL10 that have been described to play a role in the pathogenesis of marginal zone lymphomas in general are virtually absent in primary cutaneous marginal zone lymphomas, whereas the translocation $\mathrm{t}(14 ; 18)$ involving MALT1 appears to be quite common. ${ }^{3,10-15}$

Clinically, primary cutaneous marginal zone lymphomas present with solitary or multifocal red nodules or plaques that develop preferentially on the trunk and upper extremities. Relapses that can arise at the same or a different anatomic site occur in $\sim 50 \%$ of cases. Irrespective of the occurrence of relapses, primary cutaneous marginal zone lymphomas have an indolent clinical course with a 5-year survival rate of $>95 \%$. Extracutaneous manifestation of primary cutaneous marginal zone lymphomas is infrequently encountered ( $<10 \%$ of cases). This scenario raises the question of a secondary skin involvement by an originally noncutaneous marginal zone lymphoma. ${ }^{2,6}$

Given some recent reports that particular subsets of marginal zone lymphomas, specifically epidural and ocular marginal zone lymphomas, ${ }^{16-19}$ show increased frequencies of IgG4 expression and based on the known fact that primary cutaneous marginal zone lymphomas predominantly express IgG in contrast to other marginal zone lymphomas, we were interested to study the prevalence of IgG4 expression in primary cutaneous marginal zone lymphomas in comparison with various noncutaneous marginal zone lymphomas. The concept of IgG4-related diseases has attracted major attention in recent years comprising an ever increasing amount of manifestations of various organs (autoimmune pancreatitis type 1, Mikulicz's disease, Kuttner's tumor, and retroperitoneal fibrosis). ${ }^{20}$ However, a clear link between IgG4related diseases and the development of malignant lymphomas, specifically marginal zone lymphomas, has not been established so far.

To gain more insights into the relation between IgG4-related diseases and marginal zone lymphomas, we examined 169 marginal zone lymphomas with plasmacytic differentiation of various localizations for IgG4 expression, with special emphasis on primary cutaneous marginal zone lymphomas. Unexpectedly, although noncutaneous marginal zone lymphomas appeared to be almost negative, we observed IgG4 expression in 39\% of primary cutaneous marginal zone lymphomas, the highest rate of IgG4-positive cases in a particular subgroup of marginal zone lymphomas.

\section{Materials and methods}

\section{Case Selection}

We analyzed paraffin-embedded tumor specimens from 169 marginal zone lymphomas from the files of the Institute of Pathology, University of Würzburg, that were selected based on the presence of a distinct plasmacytic differentiation and light chain restriction as well as the availability of sufficient and well-preserved material. Primary sites included skin (49), stomach (21), salivary gland (20), lymph node (20), intestine (12), eye (12), lung (12), soft tissue (11), breast (3), oropharynx/larynx (3), tonsil (3), thymus (2), and thyroid gland (1). All cases were reviewed with sections stained with hematoxylin and eosin, Giemsa, and periodic acid Schiff, and an appropriate panel of immunohistochemical stains to establish a diagnosis according to the WHO classification. ${ }^{1}$ All cases were reviewed independently by two expert hematopathologists (AR and EG) and cases without consensus were excluded from the study. Approval for the entire study was obtained from the ethics committee, Medical Faculty, University of Wuerzburg, Germany.

\section{Immunohistochemistry}

Using conventional immunohistochemical single stains according to established protocols (Advance HRP Detection kit, K4068 from Dako), we investigated the expression of the immunoglobulin light chains $\kappa$ and $\lambda$ as well as the immunoglobulin heavy chains IgA, IgE, IgG, IgG4, and IgM in the marginal zone lymphoma specimens. The ratio of $\mathrm{B}$ and $\mathrm{T}$ cells in the infiltrates was estimated by CD20 and CD5 
staining and germinal center formation was highlighted by CD10 and BCL6 staining. In addition, T cells were further characterized for expression of CD4, CD8, FoxP3, and PD1. The sources of primary antibodies and their dilutions are listed in Table 1.

\section{Evaluation Criteria}

In a first step, immunoglobulin heavy chain expression (IgA, IgE, IgG, IgG4, and IgM) was determined in the light chain-restricted tumor cell population. In general, the determination of the heavy chain expression was self-evident, as $>75 \%$ of plasmacytic cells expressed one particular heavy chain, with only few intermingled cells showing expression for each of the remaining heavy chains. Cases without clearcut light or heavy chain expression were excluded from the study. In IgG-positive marginal zone lymphomas, the absolute numbers of IgG- and IgG4-positive cells were scored in five high-power fields and the ratio of IgG4/IgG was calculated. The required ratio to consider a case positive for IgG4 was an $\operatorname{IgG} 4 / \operatorname{IgG}$ ratio $>70 \%$.

In addition, primary cutaneous marginal zone lymphomas were categorized according to the absence or presence of residual lymph follicles with or without germinal center formation (CD10, BCL6). The accompanying T-cell infiltrate in primary cutaneous marginal zone lymphomas was highlighted by CD5 staining and the content of T cells among the whole lymphoid infiltrate was estimated in relation to CD20 positive B cells and categorized as low $(<20 \%)$, intermediate $(20-50 \%)$, or high ( $>50 \%$ ). Moreover, the ratio of CD4/CD8 positive $\mathrm{T}$ - cells (categories $>3: 1$ and $<3: 1$ ) and the percentage of FoxP3- and PD1-positive $\mathrm{T}$ cells among all $\mathrm{T}$ cells (categories $<1 \%, 1-5 \%, 5-10 \%$, and $>10 \%$ ) was analyzed.

\section{Statistical Analysis}

Statistical analyses were performed using the $\chi^{2}$ test. The $P$-values of $<0.05$ were considered statistically significant.

Table 1 Sources and dilutions of primary antibodies

\begin{tabular}{lllcc}
\hline Antibody & Source & Clone & Dilution & Preparation \\
\hline IgA & Biozol & Polyclonal & $1: 20000$ & CS 6.0 \\
IgM & Dako & Polyclonal & $1: 20000$ & CS 6.0 \\
IgG & Epitomics & EPR4422 & $1: 500$ & CS 6.0 \\
IgG4 & Invitrogen & HP6025 & $1: 8000$ & TR 6.1 \\
IgE & Dako & Polyclonal & $1: 2000$ & CS 6.0 \\
$\kappa$ & Dako & Polyclonal & $1: 80000$ & CS 6.0 \\
$\lambda$ & Dako & Polyclonal & $1: 80000$ & CS 6.0 \\
CD20 & Dako & L26 & $1: 500$ & CS 6.0 \\
CD5 & Novocastra & 4C7 & $1: 500$ & CS 6.0 \\
CD4 & Novocastra & 1FG & $1: 40$ & CS 6.0 \\
CD8 & Dako & C8/144B & $1: 80$ & CS 6.0 \\
FoxP3 & Abcam & 236A/E7 & $1: 50$ & TR 6.1 \\
PD1 & Abcam & NAT & $1: 200$ & TR 6.1 \\
& & & & \\
\hline
\end{tabular}

\section{Results}

\section{Clinical Characteristics of 169 Marginal Zone Lymphomas}

The median age of the 169 marginal zone lymphoma patients involved in the study was 62 years (range 8-97 years). Patients with noncutaneous marginal zone lymphomas (mean 66 years) were older than patients with primary cutaneous marginal zone lymphomas (mean 51 years, $P$-value $=0.005$ ). There were more female than male patients among noncutaneous marginal zone lymphomas (69/120, 58\% vs 51/120, 42\%), whereas there was a male predominance among primary cutaneous marginal zone lymphomas (30/49, 61\% vs 19/49, 39\%).

\section{Immunoglobulin Heavy Chain and Light Chain Expression}

The results of the immunoglobulin light and heavy chain expression of all 169 marginal zone lymphomas are detailed in Table 2.

Regarding the immunoglobulin light chain expression, there were no significant differences between non-cutaneous marginal zone lymphomas and primary cutaneous marginal zone lymphomas, both showing $\kappa$-light chain restriction in more than two thirds of cases. Specifically, $75 \%$ of noncutaneous marginal zone lymphomas showed positivity for $\kappa(90 / 120)$ and $23 \%$ for $\lambda(28 / 120)$. Two cases revealed a bispecific expression pattern proven by immunofluorescence double staining (data not shown). Of 49 primary cutaneous marginal zone lymphomas, $71 \%$ were monoclonal for $\kappa$ $(35 / 49)$ and $29 \%$ for $\lambda(14 / 35)$. More specifically, out of $35 \kappa$-positive cases, 22 showed a $\kappa / \lambda$ ratio of more than 10:1 and 13 cases of more than 20:1. Among $\lambda$-positive cases, 5/14 showed a $\lambda / \kappa$ ratio of more than 10:1, 8/14 of more than 20:1, and only one case of more than 5:1. By molecular clonality analysis, 12/19 investigated IgG4 + primary cutaneous marginal zone lymphomas showed clonal and 7/19 oligo-/polyclonal IgH rearrangements (data not shown).

A marked difference in the distribution of the immunoglobulin heavy chain expression was observed between noncutaneous marginal zone lymphomas and primary cutaneous marginal zone lymphomas. Of the 120 noncutaneous marginal zone lymphomas, $13 \%$ were positive for IgA (15/120), 9\% positive for IgG (12/120), and $78 \%$ positive for IgM (94/120), with no case showing IgE expression. In contrast, among 49 primary cutaneous marginal zone lymphomas, $72 \%$ stained positive for IgG (35/49), whereas only $12 \%$ expressed IgM (6/49) and 16\% IgA (8/49). Again, no IgE-positive case was detected. These differences were highly statistically significant $(P<0.001)$.

The major finding of our study was the frequent expression of IgG4 among primary cutaneous 
Table 2 Distribution of light and heavy chain expression in marginal zone lymphomas

\begin{tabular}{|c|c|c|c|c|c|c|c|c|c|c|c|c|c|}
\hline Localization & $\mathrm{n}$ & $\operatorname{Ig} A(\%)$ & $\kappa(\%)$ & $\lambda(\%)$ & $\operatorname{IgG}(\%)$ & $\kappa(\%)$ & $\lambda(\%)$ & $\operatorname{Ig} G 4(\%)$ & $\kappa(\%)$ & $\lambda(\%)$ & $\operatorname{Ig} M(\%)$ & $\kappa(\%)$ & $\lambda(\%)$ \\
\hline Skin & 49 & $8(16)$ & $7(88)$ & $1(12)$ & $16(33)$ & $9(56)$ & $7(44)$ & $19(39)$ & $13(68)$ & $6(32)$ & $6(12)$ & $6(100)$ & 0 \\
\hline Stomach & 21 & $3(14)$ & $1(33)$ & $2(67)$ & $4(19)$ & $2(50)$ & $2(50)$ & 0 & 0 & 0 & $14(67)$ & $12(86)$ & $2(14)$ \\
\hline Salivary gland & 20 & $1(5)$ & $1(100)$ & 0 & $2(10)$ & $2(100)$ & 0 & 0 & 0 & 0 & $17(85)$ & $16(94)$ & $1(6)$ \\
\hline Lymph node & 20 & $2(10)$ & 0 & $2(100)$ & $1(5)$ & $1(100)$ & 0 & 0 & 0 & 0 & $17(85)$ & $13(76)$ & $4(24)$ \\
\hline Intestine & 12 & $2(17)$ & $2(100)$ & 0 & $1(8)$ & $1(100)$ & 0 & 0 & 0 & 0 & $9(75)$ & $7(78)$ & $2(22)$ \\
\hline Lung & 12 & $1(8)$ & $1(100)$ & 0 & 0 & 0 & 0 & 0 & 0 & 0 & $11(92)$ & $8(73)$ & $3(27)$ \\
\hline Eye & 12 & $2(17)$ & $1(50)$ & $1(50)$ & $1(8)$ & $1(100)$ & 0 & $1(8)$ & $1(100)$ & 0 & $8(67)$ & $6(75)$ & $2(25)$ \\
\hline Soft tissue & 11 & $2(18)$ & $1(50)$ & $1(50)$ & 0 & 0 & 0 & 0 & 0 & 0 & $9(82)$ & $6(67)$ & $3(33)$ \\
\hline Breast & 3 & 0 & 0 & 0 & 0 & 0 & 0 & 0 & 0 & 0 & $3(100)$ & $3(100)$ & 0 \\
\hline Oropharynx/larynx & 3 & 0 & 0 & 0 & 0 & 0 & 0 & 0 & 0 & 0 & $3(100)$ & $2(67)$ & $1(33)$ \\
\hline Tonsil & 3 & $1(33)$ & 0 & $1(100)$ & 0 & 0 & 0 & 0 & 0 & 0 & $2(67)$ & $2(100)$ & 0 \\
\hline Thymus & 2 & 0 & 0 & 0 & $1(50)$ & 0 & $1(100)$ & 0 & 0 & 0 & $1(50)$ & $1(100)$ & 0 \\
\hline Thyroid gland & 1 & $1(100)$ & $1(100)$ & 0 & 0 & 0 & 0 & 0 & 0 & 0 & 0 & 0 & 0 \\
\hline
\end{tabular}

marginal zone lymphomas. Of all the investigated primary cutaneous marginal zone lymphomas, $39 \%$ (19/49) expressed IgG4, whereas only 1 noncutaneous marginal zone lymphoma (ocular lymphoma) out of 120 cases had IgG4 expression in the tumor cells. Thus, primary cutaneous marginal zone lymphomas appear to be the subgroup of marginal zone lymphomas with by far the most frequent expression of IgG4. This finding prompted us to perform a more detailed investigation of the clinical and morphological features of primary cutaneous marginal zone lymphomas. The absolute count for IgG4 + cells/HPF depended on the total amount of light chain-restricted plasma cells and differed from case to case, ranging from 61 to 218 IgG4 + plasma cells (mean 129) in our cases (Table 3).

\section{Morphological and Phenotypical Characteristics of Primary Cutaneous Marginal Zone Lymphomas}

A typical example of a primary cutaneous marginal zone lymphoma with plasmacytic differentiation selected for our study is shown in Figure 1. A clearcut light chain restriction as a prerequisite for inclusion in the current series and a prototype of an IgG4-expressing primary cutaneous marginal zone lymphoma is illustrated in Figure 2.

Given the high frequency of IgG4-positive primary cutaneous marginal zone lymphomas, we wanted to investigate whether this subgroup shows differences in architectural features or in the composition of the reactive T-cell infiltrate in comparison with nonIgG4 primary cutaneous marginal zone lymphomas including primary cutaneous marginal zone lymphomas expressing IgA, IgM, and IgG1-3 (defined by positivity for IgG and negativity for IgG4).

With regard to the presence or absence of lymph follicles in the infiltrate, $71 \%$ of the 49 primary cutaneous marginal zone lymphomas showed lymph follicles with germinal center formation, $14 \%$ had B-cell follicles without germinal centers, and $15 \%$ had no follicle formation at all. No differences were observed between IgG4-positive and non-IgG4 primary cutaneous marginal zone lymphomas. Similarly, these subgroups did not differ in terms of numbers and localization of plasmacytic tumor cells.

We next investigated whether the T-cell content showed any variation between primary cutaneous marginal zone lymphoma subgroups expressing different heavy chains. It is noteworthy that IgM-positive primary cutaneous marginal zone lymphomas were characterized by a low number of infiltrating $\mathrm{T}$ cells $(P<0.05)$, whereas no significant differences could be discerned between the remaining subgroups that in the majority of cases harbored $>50 \%$ T cells among all lymphoid cells (Figure 3). Moreover, all IgM-positive primary cutaneous marginal zone lymphomas displayed an increased CD4/CD8 ratio $(>3: 1)$ compared with the three other subgroups $(P<0.05$; Figure 4$)$.

Regulatory T cells stained by immunohistochemistry for FOXP3 were generally scarce in primary cutaneous marginal zone lymphomas $(<10 \%$ of all CD5-positive $\mathrm{T}$ cells) and diffusely distributed across the infiltrate. Again, IgM-positive primary cutaneous marginal zone lymphomas differed from the other subgroups by exhibiting a very low number of regulatory $\mathrm{T}$ cells $(<1 \%)$ in $50 \%$ of cases $(P<0.05$; Figure 5). The presence of PD1-positive follicular helper $\mathrm{T}$ cells that were only counted in the neoplastic infiltrate (outside the reactive follicles) showed no statistical differences between the four primary cutaneous marginal zone lymphoma subgroups (data not shown).

\section{Detailed Clinical Characteristics of Primary Cutaneous Marginal Zone Lymphomas}

From a clinical point of view, we finally wondered whether IgG4-positive primary cutaneous marginal zone lymphomas might display particular features separating them from the other primary cutaneous marginal zone lymphoma heavy chain subgroups. A detailed overview of clinical characteristics of the 
Table 3 Clinical and immunohistochemical characteristics of IgG4 + primary cutaneous marginal zone lymphomas

\begin{tabular}{|c|c|c|c|c|c|c|c|c|c|c|c|c|}
\hline Case & Site & Gender & Age & $\begin{array}{l}\operatorname{IgG4/IgG} \\
(\%) / \text { cell } \\
\text { count/HPF } \\
\text { (mean) }\end{array}$ & $\begin{array}{l}\text { Light } \\
\text { chain }\end{array}$ & Treatment & Relapse & Staging & $\begin{array}{l}\text { Borrelia } \\
\text { burgdorferi }\end{array}$ & $L D H$ & $\begin{array}{l}\text { Number of } \\
\text { lesions at } \\
\text { presentation }\end{array}$ & $\begin{array}{l}\text { Clinical } \\
\text { presentation of } \\
\text { the lesions } \\
\text { (nodule vs other) }\end{array}$ \\
\hline 1 & Neck & $\mathrm{M}$ & 51 & 99/89 & $\kappa$ & $\mathrm{E}$ & No & NEM & Negative & NA & 1 & Nodule \\
\hline 2 & $\begin{array}{l}\text { Upper } \\
\text { extremitiy }\end{array}$ & $\mathrm{M}$ & 70 & $99 / 218$ & $\lambda$ & $\mathrm{E}$ & No & NEM & NA & Elevated & 1 & Nodule \\
\hline 3 & $\begin{array}{l}\text { Lower } \\
\text { extremity }\end{array}$ & M & 32 & $98 / 124$ & $\kappa \kappa$ & E, D & Yes & NEM & NA & Normal & 1 & Nodule \\
\hline 4 & Back & $\mathrm{M}$ & 74 & $99 / 130$ & $\kappa$ & C, PD & Yes & NEM & Positive & Normal & $>2$ & Nodule \\
\hline 5 & Back & $\mathrm{F}$ & 53 & $95 / 173$ & $\lambda$ & $\mathrm{E}$ & NA & NEM & Negative & Normal & 1 & Nodule \\
\hline 6 & Back & $\mathrm{F}$ & 28 & $83 / 92$ & $\lambda$ & E, D & yes & NEM & Negative & Normal & 1 & Nodule \\
\hline 7 & Back & M & 26 & $78 / 75$ & $\kappa$ & NA & NA & NA & NA & NA & 1 & Nodule \\
\hline 8 & Shoulder & M & 46 & $96 / 210$ & $\kappa$ & $\mathrm{C}, \mathrm{R}, \mathrm{PD}$ & NA & NEM & NA & Normal & $>2$ & Other \\
\hline 9 & $\begin{array}{l}\text { Upper } \\
\text { extremitiy }\end{array}$ & $\mathrm{F}$ & 75 & $90 / 110$ & $\lambda$ & $\mathrm{E}, \mathrm{R}$ & Yes & NEM & NA & Normal & 1 & Nodule \\
\hline 10 & Shoulder & $\mathrm{M}$ & 69 & $77 / 124$ & $\lambda$ & $\mathrm{E}$ & Yes & NA & NA & NA & NA & Nodule \\
\hline 11 & Back & $\mathrm{F}$ & 58 & $83 / 110$ & $\kappa$ & $\mathrm{R}$ & No & NEM & NA & Normal & 1 & Other \\
\hline 12 & Shoulder & M & 74 & $76 / 108$ & $\lambda$ & $\mathrm{E}$ & No & NEM & NA & Normal & 1 & Other \\
\hline 13 & Back & M & 29 & $95 / 131$ & $\kappa$ & $\mathrm{E}$ & Yes & NEM & Negative & Normal & $>2$ & Nodule \\
\hline 14 & NA & $\mathrm{M}$ & 36 & $84 / 84$ & $\kappa$ & NA & Yes & NA & NA & NA & 1 & Nodule \\
\hline 15 & Back & M & 40 & 99/177 & $\kappa$ & $\mathrm{E}, \mathrm{R}$ & No & NEM & NA & normal & 1 & Nodule \\
\hline 16 & Head & $\mathrm{F}$ & 55 & $90 / 100$ & $\kappa$ & NA & Yes & NA & NA & NA & 1 & Nodule \\
\hline 17 & $\begin{array}{l}\text { Upper } \\
\text { extremitiy }\end{array}$ & M & 37 & 96/185 & $\kappa$ & $\mathrm{R}$ & No & NEM & NA & Normal & 1 & Nodule \\
\hline 18 & Shoulder & $\mathrm{M}$ & 80 & $99 / 111$ & $\kappa$ & NA & NA & NA & NA & NA & 1 & Nodule \\
\hline 19 & $\begin{array}{l}\text { Upper } \\
\text { extremitiy }\end{array}$ & $\mathrm{F}$ & 49 & $80 / 117$ & $\kappa$ & NA & Yes & NA & NA & NA & 1 & Other \\
\hline
\end{tabular}

Abbreviations: C, chemotherapy; D, doxycyclin; E, excision; F, female; M, male; NA, not available; NEM, no extracutaneous manifestation; $\mathrm{PD}$, progressive disease; $\mathrm{R}$, radiation.

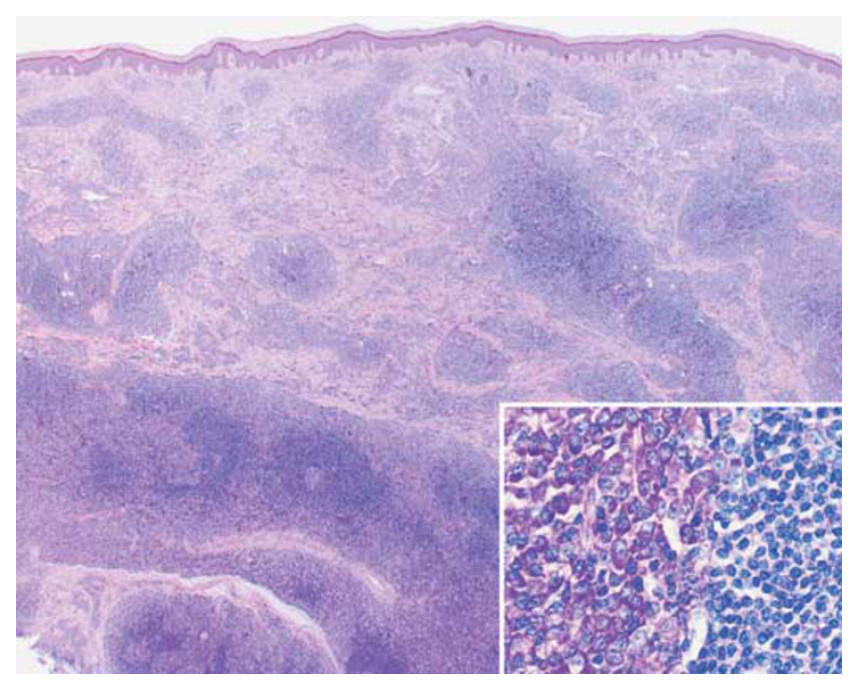

Figure 1 Typical morphological pattern of primary cutaneous marginal zone lymphoma (case 2) showing a diffuse and nodular infiltrate involving the whole dermis but sparing the epidermis. The infiltrate consists of reactive lymph follicles surrounded by broadened marginal zones. The inset highlights clusters of tumor cells with plasmacytic differentiation (Giemsa).

IgG4-positive primary cutaneous marginal zone lymphomas is provided in Table 3. Overall, the median age of the primary cutaneous marginal zone lymphoma patients was 51 years, and more men than women were affected. IgM-positive primary cutaneous marginal zone lymphoma patients tended to be slightly older, although this difference was not statistically significant (IgA: mean 49 years, IgG1-3: mean 48 years, IgG4: mean 52 years, IgM: mean 63 years). Although there was a male preponderance in primary cutaneous marginal zone lymphoma patients expressing IgA (3:1), IgG4 (2.2:1), and IgM (2:1), females were slightly, but not significantly, more affected among IgG1-3-positive primary cutaneous marginal zone lymphomas (1.3:1).

Primary cutaneous marginal zone lymphomas in our series manifested in the back $(n=15)$, upper extremities $(n=10)$, shoulder $(n=9)$, abdomen $(n=3)$, head $(n=3)$, neck $(n=2)$, lower extremities $(n=2)$, and genital tract $(n=1)$. In four cases, the primary manifestation could not be determined. In all, 40/49 primary cutaneous marginal zone lymphomas appeared as a solitary manifestation and 9/49 as multiple manifestations. With regard to localization and solitary vs multiple lesions, no statistical differences could be observed between the four primary cutaneous marginal zone lymphoma subgroups. Additional clinical information was available from 32 of 49 primary cutaneous marginal zone lymphomas patients. As for the rate of relapses (available from 32 patients), staging results (available from 30 patients), and serology of Borrelia burgdorferi (available from 9 patients), no differences were seen among the primary cutaneous marginal zone lymphoma heavy chain subgroups. Only one primary cutaneous marginal zone lymphoma (IgG1-3) 

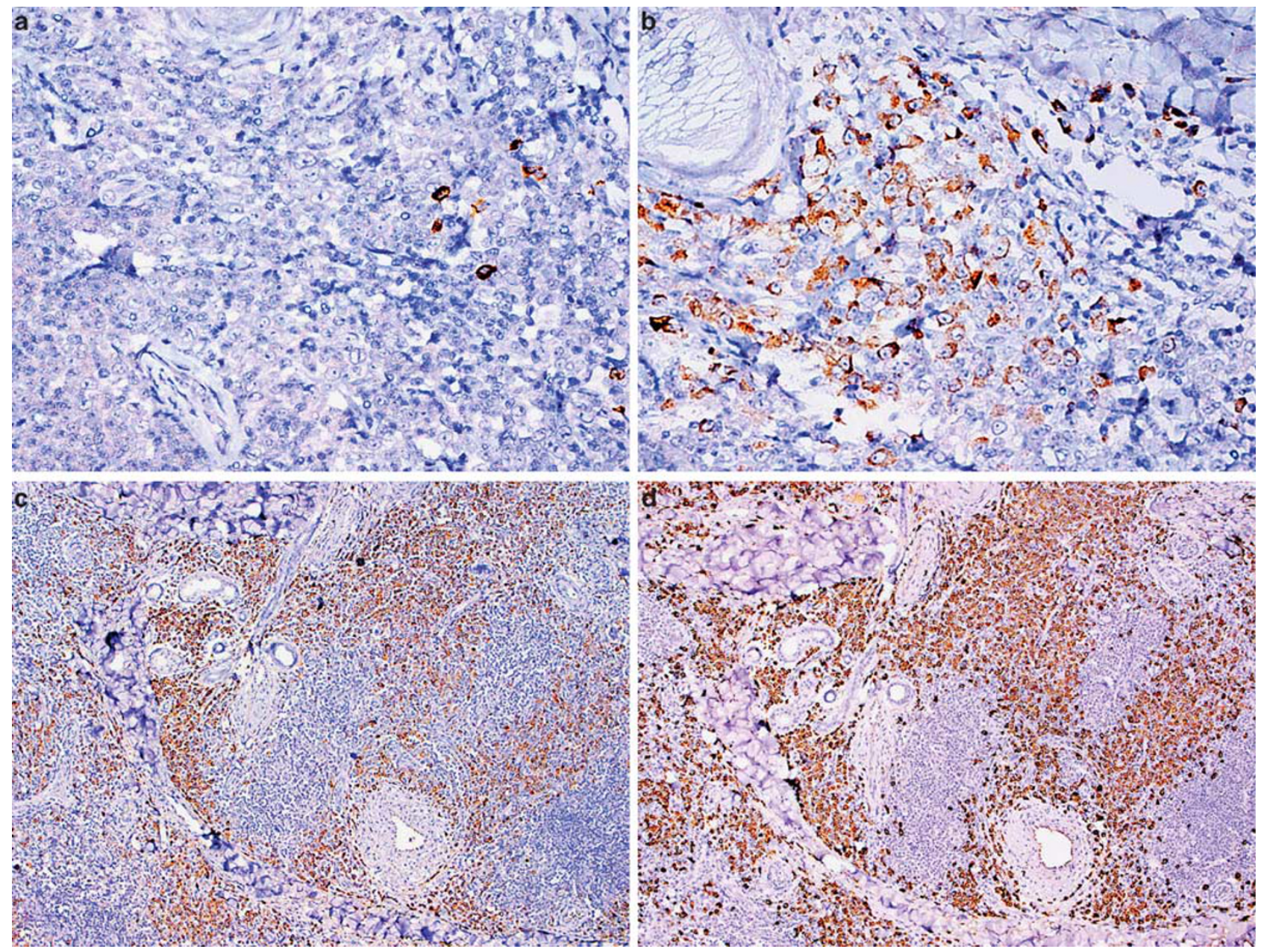

Figure 2 IgG4-positive primary cutaneous marginal zone lymphoma (case 2). The plasmacytic tumor cells of a primary cutaneous marginal zone lymphoma lack $\kappa$ expression (a) and show monoclonal $\lambda$ light chain restriction (b). They express IgG (c) and IgG4 (d).

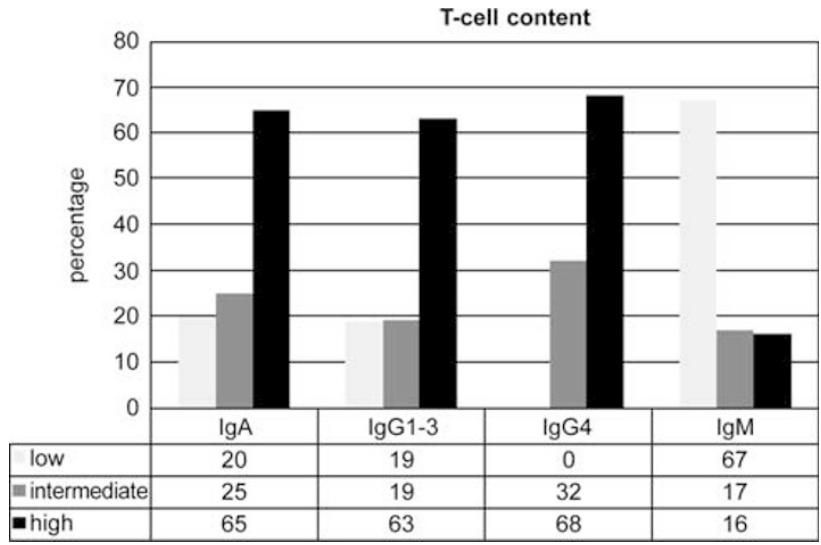

Figure 3 Variation of T-cell content between primary cutaneous marginal zone lymphoma subgroups expressing different immunoglobulin heavy chains.

showed a secondary lymph node involvement, whereas in 30/32 primary cutaneous marginal zone lymphoma patients, extracutaneous manifestations/ secondary skin involvement of a primary, noncuta-

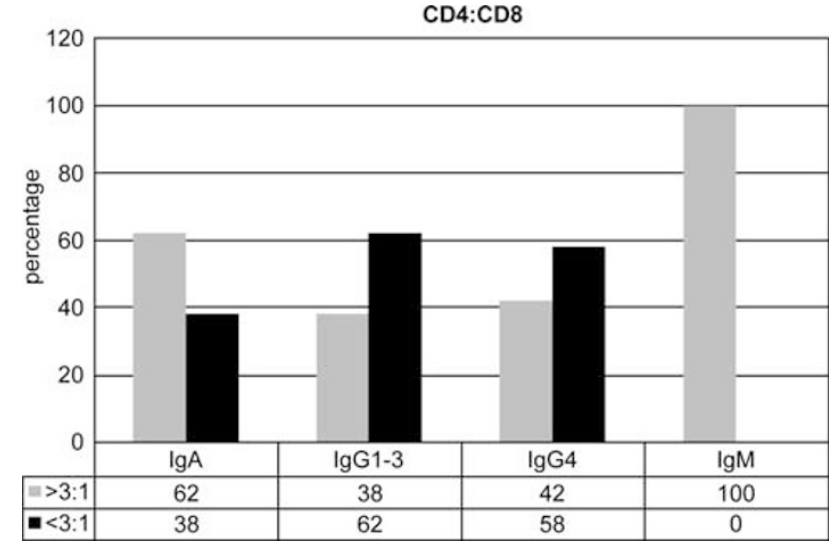

Figure 4 CD4/CD8 ratio in primary cutaneous marginal zone lymphoma subgroups expressing different immunoglobulin heavy chains.

neous marginal zone lymphomas could be ruled out by staging procedures. Finally, there was no evidence of an associated IgG4-related disease in any of the patients. 


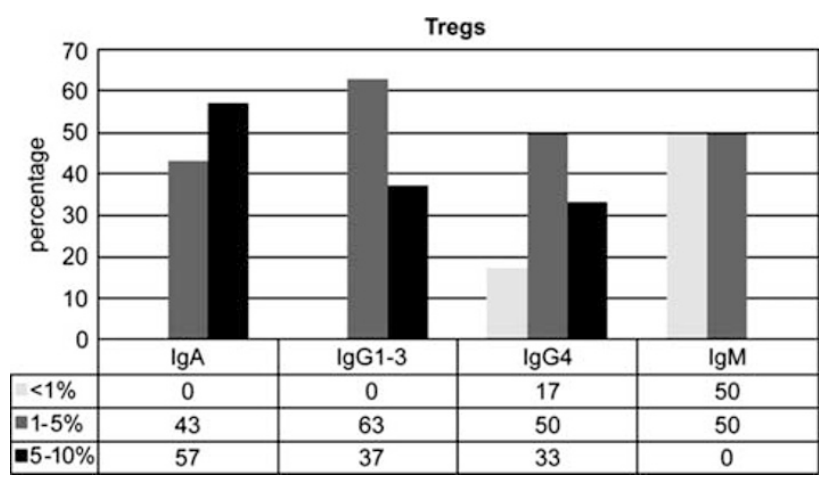

Figure 5 Variation of regulatory $\mathrm{T}$ cell (Treg) content between primary cutaneous marginal zone lymphoma subgroups expressing different immunoglobulin heavy chains.

\section{Discussion}

Primary cutaneous marginal zone lymphomas differ from noncutaneous marginal zone lymphomas in various aspects with respect to biological behavior, genetic features, and immunophenotypic presentation. Whereas primary cutaneous marginal zone lymphomas exhibit a preferential expression of $\operatorname{IgA}$ and $\operatorname{IgG}$, noncutaneous marginal zone lymphomas display a predominant IgM expression. , $^{7,}$

Recent studies suggest two major biological subsets within primary cutaneous marginal zone lymphomas, namely one subset that expresses class-switched immunoglobulins (IgA, $\operatorname{IgG}$, and IgE), whereas the other, rarer subtype expresses nonclass-switched immunoglobulins (IgM).7,9 This subdivision appears to correlate with profound differences in the composition of the microenvironment and cytokine/chemokine profiles. Specifically, the IgM-positive group develops in a preferential Th1 cytokine background and shows increased levels of CXCR3 expression, analogous to the scenario in most of the noncutaneous marginal zone lymphomas. In contrast, the class-switched group is characterized by a dominant Th2 cytokine profile. Our study shows only a low number of infiltrating $\mathrm{T}$ cells in IgM-positive primary cutaneous marginal zone lymphomas, in contrast to the other primary cutaneous marginal zone lymphomas in which the tumor cell population is accompanied by an abundant T-cell infiltrate. This finding is in line with a recent report by Edinger et $a l^{7}$ who also detected fewer infiltrating $\mathrm{T}$ cells in IgM-positive primary cutaneous marginal zone lymphomas. Moreover, our investigation revealed a CD4/CD8 ratio in IgM-positive primary cutaneous marginal zone lymphomas that was skewed toward an increased number of CD4-positive $\mathrm{T}$ cells. The percentage of regulatory $\mathrm{T}$ cells among all $\mathrm{T}$ cells present in a given infiltrate was also decreased in IgM-positive primary cutaneous marginal zone lymphomas in our study. Taken together, these results support the notion that class-switched and nonclass-switched primary cutaneous marginal zone lymphomas have distinct biological features. It has been postulated that IgM-positive primary cutaneous marginal zone lymphomas may be more frequently associated with a Borrelia burgdorferi infection. ${ }^{9}$ In our series, however, the two primary cutaneous marginal zone lymphoma patients (out of nine patients with available information) with positive serology for Borrelia burgdorferi did not express IgM in the tumor cells.

The major and unexpected finding of our study is the high frequency of IgG4 expression in primary cutaneous marginal zone lymphomas. Although IgG4 expression has been described in a single case of a nodal marginal zone lymphoma, ${ }^{21}$ few cases of ocular adnexae marginal zone lymphomas, ${ }^{17-19,22}$ as well as in a subset of marginal zone lymphomas of the dura, ${ }^{16}$ the rate of $39 \%$ IgG4-positive cases among our primary cutaneous marginal zone lymphomas is striking. Only two IgG4-rearranged primary cutaneous marginal zone lymphomas, identified by RT-PCR, were previously reported. ${ }^{9}$

As morphologic and environmental features did not differ between IgG4-positive primary cutaneous marginal zone lymphomas and the remaining class-switched cases in our series, the pathogenetic consequences of IgG4 expression remain unclear at present. Although few ocular marginal zone lymphomas may show an association with an underlying IgG4-related disease, ${ }^{17,23}$ there is little evidence that this is the case for dural marginal zone lymphomas in the published series. ${ }^{16}$ Similarly, it is very unlikely that IgG4-related disease is associated with the respective subgroup of primary cutaneous marginal zone lymphomas in our cohort. Although formally serum levels of IgG4 had not been measured and staging data were incomplete in some patients, the available clinical information showed no evidence of IgG4-related disease in any of the patients (mean follow-up: 3 years). Moreover, 9/19 patients with an IgG4-positive primary cutaneous marginal zone lymphomas were $<50$ years old, an age group that is unlikely to have IgG4-related disease.

Recently, IgG4-related skin manifestations have been reported. ${ }^{24-29}$ In the largest and bestcharacterized published series of 10 patients with IgG4-related skin disease, ${ }^{24} 8 / 10$ patients were $>50$ years old, in $8 / 10$ patients extracutaneous manifestations of IgG4-related disease could be proven (lymph node, lacrimal and/or salivary gland, and kidney), and all 10 patients showed an elevated serum IgG4 level. Regarding the localization of IgG4-related skin disease that generally present on the head and neck, particularly cheek, periauricular, and mandible regions, our cases of IgG4 + primary cutaneous marginal zone lymphomas in contrast showed preferential manifestations on the back, shoulders, and upper extremities. In addition, typical histopathological characteristics of IgG4-related disease, such as fibrosclerosis, obliterative phlebitis, or an increased 
polyclonal background infiltrate of IgG4-positive plasma cells, could not be demonstrated in our primary cutaneous marginal zone lymphoma cases. Furthermore, Sato et $a l^{24}$ reported on high numbers of eosinophils in the IgG4-related skin infiltrates, a feature that was not present in our IgG4 + primary cutaneous marginal zone lymphoma cases.

Although IgG4-related disease is a polyclonal disease, IgG4 + primary cutaneous marginal zone lymphomas are clonal proliferations with light chain restriction. Clonality and/or light chain restriction have not been detected so far in four recent publications ${ }^{24-26,30}$ dealing with IgG4-related skin disease.

Physiologically, an IgG4 immune response is triggered by a long-lasting or repeated stimulation by antigen involving a modified Th2 response in which regulatory $\mathrm{T}$ cells play a dominant role. Specifically, regulatory $\mathrm{T}$ cell-associated cytokines IL-10 and IL-21 are supposed to drive the class switch in B cells to IgG4 and their differentiation into IgG4-secreting plasma cells. ${ }^{31,32}$ In this context, it was intriguing to study the abundance of regulatory $\mathrm{T}$ cells in IgG4-positive primary cutaneous marginal zone lymphomas in comparison with nonIgG4 primary cutaneous marginal zone lymphomas. The finding that the content of FOXP3-positive regulatory T cells did not differ among IgA-, IgG1-3-, and IgG4-positive primary cutaneous marginal zone lymphomas, whereas it was significantly lower in IgM-positive primary cutaneous marginal zone lymphomas in our series, has several implications. It may be postulated that the skin (and potentially the dura) may be more susceptible to developing initial, polyclonal IgG4 immune responses compared with other organs. These may eventually transform into monoclonal, IgG4-positive primary cutaneous marginal zone lymphomas during the course of the disease. Hence, it might be highly relevant to investigate IgG4-associated immune responses of the skin in more detail, as this could provide important clues for the initiating steps of lymphoma development. This, however, will not be trivial, as none of our specimens contained an increased number of residual polyclonal IgG4-positive plasma cells and elevated numbers of regulatory $\mathrm{T}$ cells as a prerequisite for an underlying IgG4 immune response. The fact that the amount of FOXP3-positive regulatory $\mathrm{T}$ cells is not increased in fully developed IgG4-positive primary cutaneous marginal zone lymphomas suggests that they are dispensable for maintaining the growth of the lymphoma. In contrast, there is good evidence that a high number of FOXP3-positive regulatory T cells in hematological malignancies suppresses tumor growth by various mechanisms, thus showing an inverse correlation with outcome. ${ }^{33,34}$ The decreased number of regulatory T cells in IgM-positive primary cutaneous marginal zone lymphomas compared with the other primary cutaneous marginal zone lymphomas in our series might therefore contribute to the inferior clinical outcome of IgM-positive primary cutaneous marginal zone lymphomas that has been suggested in the report by Edinger et al. ${ }^{7}$ Although the number of IgM-positive primary cutaneous marginal zone lymphomas was low in our cohort, all four patients with available clinical data in this group showed a higher tendency toward relapses (data not shown).

In conclusion, we herein show that primary cutaneous marginal zone lymphomas with plasmacytic differentiation have frequent IgG4 expression (39\% of 49 analyzed cases), suggesting a novel important pathogenetic feature that separates primary cutaneous marginal zone lymphomas from noncutaneous marginal zone lymphomas. Moreover, from a diagnostic point of view, the expression of IgG4 in a marginal zone lymphoma infiltrating the skin may be viewed as strong evidence of the presence of a genuine, primary cutaneous marginal zone lymphoma. Even though the number of IgG4-positive primary cutaneous marginal zone lymphomas with available clinical data are limited in our series, the fact that none of these patients had signs of extracutaneous spread in staging procedures suggests an excellent prognosis. If proven in a larger clinical cohort, IgG4 expression may represent a novel discriminating diagnostic aid that could save IgG4-positive primary cutaneous marginal zone lymphoma patients from aggressive therapeutic approaches as well as from cumbersome and costly staging procedures in the future.

\section{Disclosure/conflict of interest}

The authors declare no conflict of interest.

\section{References}

1 Swerdlow SH, Campo E, Harris NL, et al. WHO Classification of Tumours of Haematopoietic and Lymphoid Tissues Vol. 2 (IARC: Lyon, 2008).

2 Dalle S, Thomas L, Balme B, et al. Primary cutaneous marginal zone lymphoma. Crit Rev Oncol Hematol 2010;74:156-162.

3 Takino H, Li C, Hu S, et al. Primary cutaneous marginal zone B-cell lymphoma: a molecular and clinicopathological study of cases from Asia, Germany, and the United States. Mod Pathol 2008;21:1517-1526.

4 May SA, Netto G, Domiati-Saad R, et al. Cutaneous lymphoid hyperplasia and marginal zone B-cell lymphoma following vaccination. J Am Acad Dermatol 2005;53:512-516.

5 Breton AL, Poulalhon N, Balme B, et al. Primary cutaneous marginal zone lymphoma as a complication of radiation therapy: case report and review. Dermatol Online J 2010;16:6.

6 Kempf W, Denisjuk N, Kerl K, et al. Primary cutaneous B-cell lymphomas. J Dtsch Dermatol Ges 2012;10: 12-22; quiz 3.

7 Edinger JT, Kant JA, Swerdlow SH. Cutaneous marginal zone lymphomas have distinctive features and 
include 2 subsets. Am J Surg Pathol 2010;34: 1830-1841.

8 Ferenczi K. Primary cutaneous marginal zone B-cell lymphomas: are they different from other extranodal marginal zone B-cell lymphomas? J Cutan Pathol 2009;36:715-717.

9 van Maldegem F, van Dijk R, Wormhoudt TA, et al. The majority of cutaneous marginal zone B-cell lymphomas expresses class-switched immunoglobulins and develops in a T-helper type 2 inflammatory environment. Blood 2008;112:3355-3361.

10 Gronbaek K, Ralfkiaer E, Kalla J, et al. Infrequent somatic Fas mutations but no evidence of Bcl10 mutations or $\mathrm{t}(11 ; 18)$ in primary cutaneous MALTtype lymphoma. J Pathol 2003;201:134-140.

11 Hallermann C, Kaune KM, Gesk S, et al. Molecular cytogenetic analysis of chromosomal breakpoints in the IGH, MYC, BCL6, and MALT1 gene loci in primary cutaneous B-cell lymphomas. J Invest Dermatol 2004;123:213-219.

12 Espinet B, Gallardo F, Pujol RM, et al. Absence of MALT1 translocations in primary cutaneous marginal zone B-cell lymphoma. Haematologica 2004;89: ELT14.

13 Streubel B, Lamprecht A, Dierlamm J, et al. $\mathrm{T}(14 ; 18)(\mathrm{q} 32 ; \mathrm{q} 21)$ involving IGH and MALT1 is a frequent chromosomal aberration in MALT lymphoma. Blood 2003;101:2335-2339.

14 Streubel B, Simonitsch-Klupp I, Mullauer L, et al. Variable frequencies of MALT lymphoma-associated genetic aberrations in MALT lymphomas of different sites. Leukemia 2004;18:1722-1726.

15 Streubel B, Vinatzer U, Lamprecht A, et al. $\mathrm{T}(3 ; 14)(\mathrm{p} 14.1 ; \mathrm{q} 32)$ involving IGH and FOXP1 is a novel recurrent chromosomal aberration in MALT lymphoma. Leukemia 2005;19:652-658.

16 Venkataraman G, Rizzo KA, Chavez JJ, et al. Marginal zone lymphomas involving meningeal dura: possible link to IgG4-related diseases. Mod Pathol 2011;24: 355-366.

17 Cheuk W, Yuen HK, Chan AC, et al. Ocular adnexal lymphoma associated with IgG4 + chronic sclerosing dacryoadenitis: a previously undescribed complication of IgG4-related sclerosing disease. Am J Surg Pathol 2008;32:1159-1167.

18 Sato Y, Ohshima K, Ichimura K, et al. Ocular adnexal IgG4-related disease has uniform clinicopathology. Pathol Int 2008;58:465-470.

19 Sato Y, Ohshima K, Takata K, et al. Ocular adnexal IgG4-producing mucosa-associated lymphoid tissue lymphoma mimicking IgG4-related disease. J Clin Exp Hematop 2012;52:51-55.
20 Stone JH, Zen Y, Deshpande V. IgG4-related disease. N Engl J Med 2012;366:539-551.

21 Sato Y, Takata K, Ichimura K, et al. IgG4-producing marginal zone B-cell lymphoma. Int J Hematol 2008;88:428-433.

22 Sato Y, Notohara K, Kojima M, et al. IgG4-related disease: historical overview and pathology of hematological disorders. Pathol Int 2010;60:247-258.

23 Cheuk W, Yuen HK, Chan JK. Complication of IgG4-related chronic sclerosing dacryoadenitis by lymphoma. Arch Ophthalmol 2008;126:1170 author reply.

24 Sato Y, Takeuchi M, Takata K, et al. Clinicopathologic analysis of IgG4-related skin disease. Mod Pathol 2013;26:523-532.

25 Ikeda T, Oka M, Shimizu H, et al. IgG4-related skin manifestations in patients with IgG4-related disease. Eur J Dermatol 2013;23:241-245.

26 Ingen-Housz-Oro S, Ortonne N, Elhai $\mathrm{M}$, et al. IgG4-related skin disease successfully treated by thalidomide: a report of 2 cases with emphasis on pathological aspects. JAMA Dermatol 2013; doi:10.1001/ jamadermatol.2013.2625 [e-pub ahead of print].

27 Kato K, Satoh T, Tanaka-Fujimoto T, et al. IgG4-positive cells in skin lesions of cutaneous and systemic plasmacytosis. Eur J Dermatol 2013;23:255-256.

28 Khosroshahi A, Carruthers MD, Deshpande V, et al. Cutaneous immunoglobulin G4-related systemic disease. Am J Med 2011;124:e7-e8.

29 Cheuk W, Lee KC, Chong LY, et al. IgG4-related Sclerosing disease: a potential new etiology of cutaneous pseudolymphoma. Am J Surg Pathol 2009;33: 1713-1719.

$30 \mathrm{~J}$ Godfrey Heathcote NMW, Sutton Evelyn D, Valenzuela Alejandra A. IgG4-related disease manifesting as sclerosing orbital inflammation and cutaneous pseudolymphoma with crystal-storing histiocytosis. Diagn Pathol 2013;19:147-150.

31 Aalberse RC, Stapel SO, Schuurman J, et al. Immunoglobulin G4: an odd antibody. Clin Exp Allergy 2009;39:469-477.

32 Nirula A, Glaser SM, Kalled SL, et al. What is IgG4? A review of the biology of a unique immunoglobulin subtype. Curr Opin Rheumatol 2011;23:119-124.

33 Kelley TW, Parker CJ. CD4 (+)CD25 (+)Foxp3 (+) regulatory $\mathrm{T}$ cells and hematologic malignancies. Front Biosci (Schol Ed) 2010;2:980-992.

34 Tzankov A, Meier C, Hirschmann P, et al. Correlation of high numbers of intratumoral FOXP3 + regulatory T cells with improved survival in germinal center-like diffuse large B-cell lymphoma, follicular lymphoma and classical Hodgkin's lymphoma. Haematologica 2008;93:193-200. 